\title{
The rotation of Galaxy Clusters
}

\author{
Hrant M. TOVMassian \\ 377, W.California, 30, Glendale, CA, 91203, USA \\ htovmas@gmail.com \\ Octuber 14, 2015
}

\begin{abstract}
The method for detection of the galaxy cluster rotation based on the study of distribution of member galaxies with velocities lower and higher of the cluster mean velocity over the cluster image is proposed. The search for rotation is made for flat clusters with $a / b>1.8$ and BMI type clusters which are expected to be rotating. For comparison there were studied also round clusters and clusters of NBMI type, the second by brightness galaxy in which does not differ significantly from the cluster $c D$ galaxy. Seventeen out of studied 65 clusters are found to be rotating. It was found that the detection rate is sufficiently high for flat clusters, over $60 \%$, and clusters of BMI type with dominant $c D$ galaxy, $\approx 35 \%$. The obtained results show that clusters were formed from the huge primordial gas clouds and preserved the rotation of the primordial clouds, unless they did not have mergings with other clusters and groups of galaxies, in the result of which the rotation has been prevented.
\end{abstract}

\section{INTRODUCTION}

The knowledge of the dynamical state of galaxy clusters could provide important constraints on cosmological scenarios. It is widely assumed that the dynamics of clusters is mostly governed by infall models and the theory of caustics (Reg'os \& Geller [1]; van Haarlem et al. [2]; Diaferio \& Geller [3]; Diaferio [4]; Rines et al. [5]. If galactic clusters are formed by hierarchical merging of groups of galaxies, no rotation of a cluster, as a whole, is possible. However, the formation of a cluster from primordial giant gas cloud may not be overlooked. If so, the formed cluster may preserve the rotation of the primordial gas cloud, if afterwards it have not merged with other clusters and groups. The problem of possible rotation of galaxy clusters has been discussed by many (Kalinkov [6], Gregory [7], Gregory \& Tift [8], Gregory \& Thompson [9], Materne \& Hopp [10], Materne, [11] Williams [12] Oegerly \& Hill [13], Sodre'e et al. [14], Biviano et al. [15], Den Hartog \& Katgert [16], Dupke \& Bregman [17, 18], Tovmassian [19], Burgett et al. [20], Kalinkov et al. [21], Hwang \& Lee [22]. Though indica- tions of rotation were found in some clusters $[10,16,20-22]$, the general accepted opinion is that galaxy clusters do not rotate. The sparse rotating clusters were found among arbitrarily selected cluster samples. Den Hartog \& Katgert [16] found 13 possibly rotating ones out of studied 72 clusters. Hwang \& Lee [22] detected only 13 tentatively rotating clusters among studied 899 Abell clusters.

For detection the cluster rotation Den Hartog \& Katgert [16] plotted line-of-sight velocity dispersion against the projected radial distance of the galaxy from the cluster center. Hwang \& Lee [22] fitted the observed radial velocities $v_{p}$ of the cluster galaxies with a function of position angle, $v_{p}(\theta)$. In both methods it was assumed that the observed distance of a galaxy from the cluster center is the real distance, and the galaxy observed at this position has the velocity corresponding to the rotation model. However, this approach could not be applied for all observed galaxies. Tovmassian \& Mntsakanian [23] studied 3D-distribution of galaxies in clusters and showed that the number of observed galaxies over the cluster area with certain radius is sufficiently higher 
of the number of galaxies within the sphere of the same radius. For example, the number of galaxies observed over the small central area of the cluster with radius about five times smaller of the cluster Abell radius, is by $4 \div 5$ times higher of the number of galaxies within the corresponding sphere. Most of the observed galaxies here are projected galaxies from the outer spherical shells of the cluster. Some of them could be located at the cluster border, and their rotational velocities would be significantly different from the assumed velocities. Also, it was shown [23] that on average about $25 \%$ of galaxies observed over the cluster are projected galaxies of the cluster environment that have the same velocities, as the cluster proper members. They will introduce additional errors in the analysis. It follows that the possible rotation of the cluster could hardly be revealed by the study of the correlation between the radial velocity dispersion and the projected galaxy position in the cluster.

I propose a simple method for detecting the cluster rotation, that does not depend on the projected position of galaxies in relation to the rotation axes. First, in order to minimize the influence of projected environmental galaxies on the results, it is desirable to study the possibly small central area of the cluster. I limited the radius of the studied area so in order to have not less than about 20 galaxies there. Then, I counted galaxies with velocities lower, $V_{l}$, and higher, $V_{h}$, of the mean velocity $V^{*}$ of galaxies in the studied area. If a cluster is experiencing merging, the numbers $n_{l}$ and $n_{h}$ of galaxies with velocities lower and higher of the mean velocity $V^{*}$ will sufficiently differ from each other. I assume that a cluster is in the state of merging, if numbers $n_{l}$ and $n_{h}$ differ from each other by more than 1.2 times. If a cluster is in dynamical equilibrium, the numbers of galaxies with velocities lower and higher of the cluster mean velocity will be approximately the same (the ratio of their numbers will be smaller than 1.2), at any half of the cluster image. In a rotating cluster the assumed rotation axes will pass through or be located close to the adopted cluster center. The numbers of galaxies at two sides of the rotation axes will be about the same (the ratio of numbers $<1.2$ ), but galaxies at one side of the rotation axis will have velocities higher of the mean velocity of galaxies in the studied area, and galaxies at the other side will have velocities lower of the mean velocity. However, due to interactions between close neighbors, especially in the central dense regions, there could be member galaxies with not rotational velocities. As a result, the regularities that are characteristic for a rotating cluster would be somewhat deteriorated. Environmental galaxies projected over the cluster and interlopers will mask more the effect of rotation. Anyhow, in a rotating cluster the majority of galaxies at one side of the rotation axes will move in direction opposite to the direction of movement of the majority of galaxies at the other side. We assume that the cluster is rotating, if the portion of galaxies that rotate in the cluster, constitute more than $60 \%$ of all galaxies at each side of the rotation axes. Hence, by analyzing the distribution of galaxies with velocities lower and higher of the cluster mean velocity it will be possible to detect rotating clusters.

In this paper I checked the proposed method for detection of the cluster rotation by using four samples of galaxies. The first sample consists of highly flattened ACO [24] clusters from Strubble and Ftaclas [25] with $f=a / b$ exceeding 1.8. Here $a$ and $b$ are respectively the cluster large and small axis. The high flatness could be evidence of merging of two clusters. Clusters of high flatness could also be rotating. For comparison I used the sample of round clusters with $f<1.2$ [25]. The probability of detection of rotation of such clusters is apparently smaller than in flat ones.

I used also the samples of BMI and nonBMI clusters introduced by Tovmassian \& Andernach [26], who compared the Abell number count $N_{A}$ of clusters hosted the cD galaxy (type I clusters according to Bautz \& Morgan [27]), their velocity dispersion $\sigma_{v}$, the peculiar velocity of the $\mathrm{cD}$ galaxy and the cluster $\mathrm{X}$-ray brightness with absolute $K_{s-t o t a l}$ magnitude of the $\mathrm{cD}$ galaxy, and divided the clusters into two 
types. The clusters, the $K_{s-t o t a l}$ 2MASS magnitude of the $\mathrm{CD}$ galaxy of which is by more than $1^{m}$ brighter than that of the second by brightness galaxy in the cluster, were classified as BMI. The clusters the $K_{s-t o t a l}$ magnitude of the second by brightness galaxy of which is fainter than the cD galaxy by less than $0.7^{m}$, were classified as non-BMI (NBMI) type. Tovmassian \& Andernach [26] suggested that clusters of BMI and NBMI types have different evolution histories. Clusters of BMI type evolved preferentially without merging with other clusters. Meanwhile, clusters of NBMI type experienced mergers in history. Therefore, one could expect that rotating clusters could be found among BMI clusters, whereas hardly NBMI clusters will be rotating.

\section{AnAlysis AND Results}

\section{Data}

For our study we used redshifts of cluster members from SDSS-DR9 [28] that provides uniform coverage of radial velocities of member galaxies in the whole target area. Positions of clusters and their redshifts are taken from NED. The galaxies with radial velocities within \pm 1500 $\mathrm{km} \mathrm{s}^{-1}$ from the cluster mean velocity were selected as cluster members. In order to minimize the influence of projected environmental galaxies, I collected data for possibly smaller central area of the cluster. Depending on the richness of the cluster the counts were made within area with radii from 0.25 to 0.75 Abell radius, $\left.R_{A}\right]^{1}$ of the cluster. For reliability of the obtaining results, the size of the studied area was chosen so, to have in it at least 20 galaxies with known redshifts. The compiled lists of the flat and round clusters contain 18 and 13 clusters respectively. The lists of BMI and NBMI clusters consist of 20 and 16 clusters respectively. The cluster, A1663 is included in two samples: of the round clusters and of the BMI type clusters. The cluster A2147, is also included in two sample: of the flat and BMI samples. Hence, the total number of studies

\footnotetext{
${ }^{1} R_{A}=1.7 / z \operatorname{arcmin}(29)$
}

clusters is 65 .

\section{Merging clusters}

In some clusters of all four studied samples the ratio $n_{l} / n_{h}$ of numbers of galaxies with velocities lower and higher of the mean velocity of galaxies in the studied area exceeds 1.2 or is smaller than 0.8. We assume that these clusters are in the state of merging. The results of counts in merging clusters of all four samples are presented in Table 1. In the 1-st column of Table 1 the designation of the cluster is presented. In the 2-d column the size of the studied area as part of the Abell radius $R_{A}$ is shown. The numbers $n_{l}$ and $n_{h}$ are presented in columns 3 and 4 respectively. In the last column the ratio of the numbers $n_{l} / n_{h}$ is given.

\section{Not rotating clusters}

In some other clusters of all four samples the ratio of the numbers of galaxies moving towards the observer and in opposite direction is within $0.8 \div 1.20$. These clusters are not experiencing merging with other clusters. In all of them it was not possible to determine a dividing line that could be a rotating axes. Hence, these clusters are not rotating. Their list is presented in Table 2 analogues to Table 1.

\section{Rotating clusters}

In the rest of the studied clusters of all four samples the ratios of the numbers of galaxies moving towards the observer and in opposite direction are, as in previous group of galaxies, within $0.8 \div 1.2$. For these clusters the possible rotation axis are determined. The number of galaxies at two sides of the assumed rotation axes in the studied area of each cluster is about the same, the difference being less than $20 \%$. The majority of galaxies at one side of these clusters move in one direction, and the majority of galaxies at the other side - in opposite direction, that evidences on the cluster 
rotation. The numbers of galaxies with rotational movement in these clusters is by $1.5 \div 3.3$ (with median 2.2) times higher of the number of other galaxies observed in the cluster area. Therefore, we conclude that these clusters are rotating. The examples of maps of rotating clusters are presented in Figures 1 and 2 (flat clusters), Figure 3 (round cluster), and Figures 4 and 5 (clusters of BMI type). The results of counts on rotating clusters are presented in Table 3. In consecutive columns of Table 3 the following data is presented: column 1 - the Abell designation of the cluster; column 2 - the designation of the half of the cluster area for which the information is presented (W-West, E-East, NE-North-East, etc.); columns 3 and 4 the number of galaxies with velocities respectively lower and higher of the mean velocity of galaxies in the studied area of the cluster. At the upper an lower lanes for each cluster the numbers of galaxies at corresponding areas are presented.

\section{Conclusions}

A simple method for detection of rotating clusters is proposed. The essence of the method is the counts of galaxies with velocities lower and higher of the cluster mean velocity at different halves of the cluster. The method does not depend on the distance of member galaxies along the line of sight within the cluster, that affects other methods for search for cluster rotation. The applied simple method allowed to detect 17 rotating clusters among studied 65, i.e. more than the quarter of studied clusters are rotating. Note, that the rotation may not be detected for clusters the rotation axes of which is oriented close to the line of sight. The rate of rotating clusters detection is much higher than in other attempts to find rotation (e.g. [16-18; 20-22]).

The detection rate is incomparably high for flat clusters with $f=a / b>1.8$, which were assumed to be rotating. In seven out of 18 flat clusters the numbers of galaxies moving in opposite directions significantly differ from each other. Most probably they are two clus- ters in the state of merging. Out of the rest 11 really flat clusters, seven clusters, i.e. about $64 \%$, are rotating. Meanwhile, only two rotating clusters, $\approx 15 \%$, are found among 13 round clusters. The rate of rotating cluster is also very high among clusters of BMI type, the cD galaxy in which is brighter than the second by brightness galaxy by more than 1 magnitude. These clusters preferentially did not have merging in their life, as it was suggested by Tovmassian \& Andernach [26]. Seven out of the studied $20 \mathrm{BMI}$ clusters, i.e. $35 \%$, are found to be rotating. And only one rotating cluster, i.e. $\approx 6 \%$, was found among 16 NBMI clusters, which most probably have experienced mergings in the past [26]. The single rotating NBMI cluster is A2147, which is also included in the sample of flat clusters. The high percentage of rotating clusters among clusters of BMI type proves that they are indeed systems that have not experienced mergings and preserved the rotation of primordial gas clouds from which they were formed.

The found high rate of rotating clusters support the opinion that clusters were originally formed in the rotating primordial gas cloud. Then most of them became reacher in the result of hierarchical assembly of other groups and clusters of galaxies and, as a result, lost the rotation.

\section{ACKNOWLEDGEMENTS}

This research has made use of the NASA/IPAC Extragalactic Database (NED) which is operated by the Jet Propulsion Laboratory, California Institute of Technology, under contract with the National Aeronautics and Space Administration.

\section{REFERENCES}

1. Reg'os, E., \& Geller, M. J. 1991, ApJ, 377,14 .

2. van Haarlem, M. et al. 1993, MNRAS, 264,71 .

3. Diaferio, A. \& Geller, M. J. 1997, ApJ, 481, 633. 
4. Diaferio, A. 1999, MNRAS, 309, 610.

5. Rines, K. et al. 2003, AJ, 126. 2152.

6. Kalinkov, M. 1968, DoBAN, 21, 621.

7. Gregory, S. A. 1975, ApJ, 199, 1.

8. Gregory, S. A. \& Tifft, W. G. 1976, ApJ, 205,716 .

9. Gregory, S. A. \& Thompson, L. A. 1977,. ApJ, 213, 345.

10. Materne, J., \& Hopp, U. 1983, A\&A, 124, L13.

11. Materne, J. 1984, Messenger, 37, 19.

12. Williams, B. A. 1986, ApJ, 311, 25.

13. Oegerle, W. R., \& Hill, J. M. 1992, AJ, 104, 2078.

14. Sodr'e, L., Capelato, V. H., Steiner, J.E., Proust, D., \& Mazure, A. 1992, MNRAS, 259, 233.

15. Biviano, A., Durret, F., Gerbal, D., Le Fe'vre, O., Lobo, C., Mazure, A., \& Slezak, E. 1996, A\&A, 311, 95.

16. Den Hartog, R., \& Katgert, P. 1996, MNRAS, 279, 349.

17. Dupke, R. A., \& Bregman, J. N. 2001, ApJ, 562, 266.

18. Dupke, R. A., \& Bregman, J. N. 2005, ApJS, 161, 224.

19. Tovmassian, H. M. 2002, astroph/0212110.

20. Burgett, W. S., Vick, M. M. Davis, D. S. et al. 2004, MNRAS, 352, 605.

21. Kalinkov, M., Valchanov, T., Valtchanov, I., \& Kuneva, I. 2005, MNRAS, 359, 1491.

22. Hwang, H. S, \& Lee, M. G. 2007, ApJ, $662,236$.

23. Tovmassian, H. M., \& Mnatsakanian, M. A. 2015, (in preparation).

24. Abell G. O., Corwin H. G., Jr., Olowin R. P., 1989, ApJS, 70, 138.

25. Strubble, M. F., \& Ftaclas, C. 1994, AJ, $108,1$.

26. Tovmassian, H. M., \& Andernach, H. 2012, MNRAS, 427, 2047.

27. Bautz L., Morgan W. W., 1970, ApJ, 162, L149.

28. Ahn, C. P., et al. 2012, ApJS, 203, 21'

29. Andernach, H., Waldthausen, H., \& Wielebinski, R. 1980, A\&AS, 41, 339.
Table 1: Numbers of galaxies with velocities lower, $n_{l}$, and higher, $n_{h}$, of the mean velocity $V^{*}$ of galaxies in the studied area of the cluster, and their ratio in merging clusters.

\begin{tabular}{lcccc}
\hline Abell & $r / R_{A}$ & $n_{l}$ & $n_{h}$ & $n_{l} / n_{h}$ \\
\hline Flat & & & & \\
\hline A1187 & 0.50 & 18 & 32 & 0.56 \\
A1205 & 0.50 & 27 & 16 & 1.69 \\
A1257 & 0.75 & 26 & 16 & 1.62 \\
A1371 & 0.50 & 17 & 22 & 0.77 \\
A1496 & 0.75 & 14 & 20 & 0.70 \\
A2033 & 0.50 & 19 & 25 & 0.76 \\
A2175 & 0.60 & 15 & 22 & 0.68 \\
\hline Round & & & & \\
\hline A757 & 0.50 & 14 & 18 & 0.78 \\
A1781 & 0.60 & 12 & 18 & 0.66 \\
A1890 & 0.40 & 18 & 24 & 0.75 \\
A2152 & 0.30 & 12 & 22 & 0.54 \\
A2244 & 0.50 & 20 & 16 & 1.25 \\
\hline BMI & & & & \\
\hline A655 & 0.75 & 14 & 19 & 0.74 \\
A1516 & 0.75 & 21 & 29 & 0.72 \\
A1809 & 0.75 & 15 & 21 & 0.71 \\
A1864 & 0.75 & 23 & 16 & 1.44 \\
A2067 & 0.75 & 15 & 27 & 0.66 \\
A2124 & 0.50 & 19 & 27 & 0.70 \\
\hline NBMI & & & & \\
\hline A119 & 0.75 & 33 & 26 & 1.27 \\
A1691 & 0.50 & 19 & 26 & 0.73 \\
A1991 & 0.40 & 15 & 28 & 0.62 \\
A2079 & 0.50 & 15 & 21 & 0.71 \\
\hline
\end{tabular}


Table 2: Numbers of galaxies with velocities lower, $n_{l}$, and higher, $n_{h}$, of the mean velocity $V^{*}$ of galaxies in the studied area of the cluster, and their ratio in not rotating clusters.

\begin{tabular}{|c|c|c|c|c|}
\hline Abell & $r / R_{A}$ & $n_{l}$ & $n_{h}$ & $n_{l} / n_{h}$ \\
\hline \multicolumn{5}{|l|}{ Flat } \\
\hline A295 & 0.40 & 28 & 24 & 1.17 \\
\hline A1235 & 0.50 & 14 & 17 & 0.82 \\
\hline A1346 & 0.75 & 20 & 24 & 0.83 \\
\hline A1541 & 0.50 & 24 & 21 & 1.14 \\
\hline \multicolumn{5}{|l|}{ Round } \\
\hline A744 & 0.75 & 13 & 16 & 0.81 \\
\hline 1663 & 0.50 & 21 & 23 & 0.91 \\
\hline A1750 & 0.50 & 16 & 16 & 1.00 \\
\hline A1927 & 0.75 & 12 & 12 & 1.00 \\
\hline A2149 & 0.75 & 19 & 18 & 1.06 \\
\hline A2026 & 0.75 & 19 & 18 & 1.06 \\
\hline \multicolumn{5}{|l|}{ BMI } \\
\hline A208 & 0.75 & 22 & 18 & 1.22 \\
\hline A279 & 0.50 & 33 & 34 & 0.97 \\
\hline A1302 & 0.75 & 16 & 16 & 1.00 \\
\hline A1663 & 0.50 & 21 & 23 & 0.91 \\
\hline A1925 & 0.75 & 15 & 16 & 0.94 \\
\hline A2029 & 0.50 & 27 & 28 & 0.96 \\
\hline A2244 & 0.75 & 29 & 29 & 1.00 \\
\hline \multicolumn{5}{|l|}{ NBMI } \\
\hline A279 & 0.50 & 33 & 34 & 0.97 \\
\hline A754 & 0.75 & 18 & 15 & 0.12 \\
\hline 1149 & 0.75 & 17 & 18 & 0.94 \\
\hline A1650 & 0.75 & 27 & 25 & 1.08 \\
\hline A1668 & 0.75 & 15 & 16 & 0.94 \\
\hline A1800 & 0.75 & 25 & 26 & 0.96 \\
\hline A2051 & 0.75 & 13 & 16 & 0.81 \\
\hline A2063 & 0.25 & 24 & 23 & 1.04 \\
\hline A2089 & 0.75 & 30 & 25 & 1.20 \\
\hline A2428 & 0.75 & 16 & 19 & 0.84 \\
\hline A2670 & 0.50 & 25 & 30 & 0.83 \\
\hline
\end{tabular}

Table 3: Rotating clusters.

\begin{tabular}{|c|c|c|c|c|}
\hline Abell & $r / R_{A}$ & Area & $n_{l}$ & $n_{h}$ \\
\hline \multicolumn{5}{|l|}{ Flat } \\
\hline \multirow[t]{2}{*}{ A1035 } & 0.50 & NW & 10 & 16 \\
\hline & & SW & 18 & 10 \\
\hline \multirow[t]{2}{*}{ A1225 } & 0.75 & $\mathrm{~N}$ & 12 & 4 \\
\hline & & $S$ & 4 & 13 \\
\hline \multirow[t]{2}{*}{ A1362 } & 0.75 & E & 4 & 9 \\
\hline & & W & 8 & 4 \\
\hline \multirow{2}{*}{ A2069 } & 0.75 & NW & 11 & 6 \\
\hline & & SE & 6 & 12 \\
\hline \multirow[t]{2}{*}{ A2110 } & 0.75 & $\mathrm{NE}$ & 4 & 7 \\
\hline & & SW & 8 & 4 \\
\hline \multirow[t]{2}{*}{ A2147 } & 0.25 & NW & 9 & 19 \\
\hline & & SE & 15 & 12 \\
\hline \multirow[t]{2}{*}{ A2175 } & 0.6 & NW & 11 & 7 \\
\hline & & SE & 4 & 15 \\
\hline \multirow{2}{*}{ A2197 } & 0.25 & $\mathrm{NE}$ & 5 & 11 \\
\hline & & SW & 10 & 6 \\
\hline \multicolumn{5}{|l|}{ Round } \\
\hline \multirow[t]{2}{*}{ A858 } & 0.75 & SE & 10 & 4 \\
\hline & & NW & 3 & 10 \\
\hline \multirow[t]{2}{*}{ A1238 } & 0.50 & $\mathrm{~N}$ & 15 & 5 \\
\hline & & W & 6 & 14 \\
\hline \multicolumn{5}{|l|}{ BMI } \\
\hline \multirow[t]{2}{*}{ A85 } & 0.30 & NW & 14 & 6 \\
\hline & & SE & 7 & 13 \\
\hline \multirow[t]{2}{*}{ A152 } & 0.60 & SE & 13 & 8 \\
\hline & & NW & 7 & 15 \\
\hline \multirow[t]{2}{*}{ A690 } & 0.75 & E & 8 & 11 \\
\hline & & $\mathrm{W}$ & 13 & 6 \\
\hline \multirow[t]{2}{*}{ A1651 } & 0.50 & $\mathrm{~N}$ & 9 & 14 \\
\hline & & $S$ & 16 & 8 \\
\hline \multirow[t]{2}{*}{ A1738 } & 0.75 & $\mathrm{NE}$ & 5 & 12 \\
\hline & & SW & 10 & 6 \\
\hline \multirow[t]{2}{*}{ A1795 } & 0.40 & $\mathrm{~N}$ & 15 & 5 \\
\hline & & $S$ & 7 & 13 \\
\hline \multirow[t]{2}{*}{ A1890 } & 0.50 & $\mathrm{NE}$ & 6 & 16 \\
\hline & & SW & 14 & 6 \\
\hline \multicolumn{5}{|l|}{ NBMI } \\
\hline \multirow[t]{2}{*}{ A2147 } & 0.25 & NW & 9 & 20 \\
\hline & & SE & 15 & 11 \\
\hline
\end{tabular}




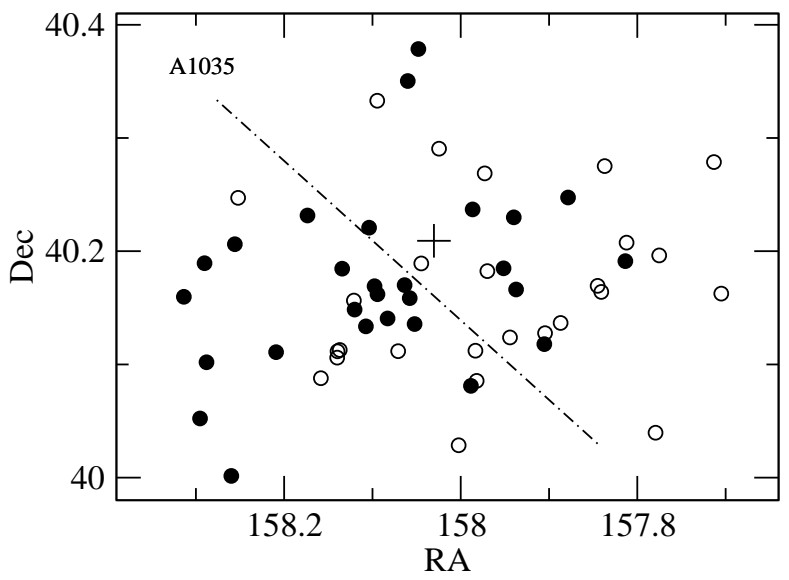

Figure 1: The map of the central region $\left(0.5 R_{A}\right)$ of the rotating cluster A1035. Filled circles are galaxies with velocities lower than the mean velocity of galaxies in the studied central area of the cluster. Open circles are galaxies with velocities higher than the mean velocity. Cross is the cluster center according to NED. Dash-dotted line is the assumed rotation axes. The same marking is used for other maps. 


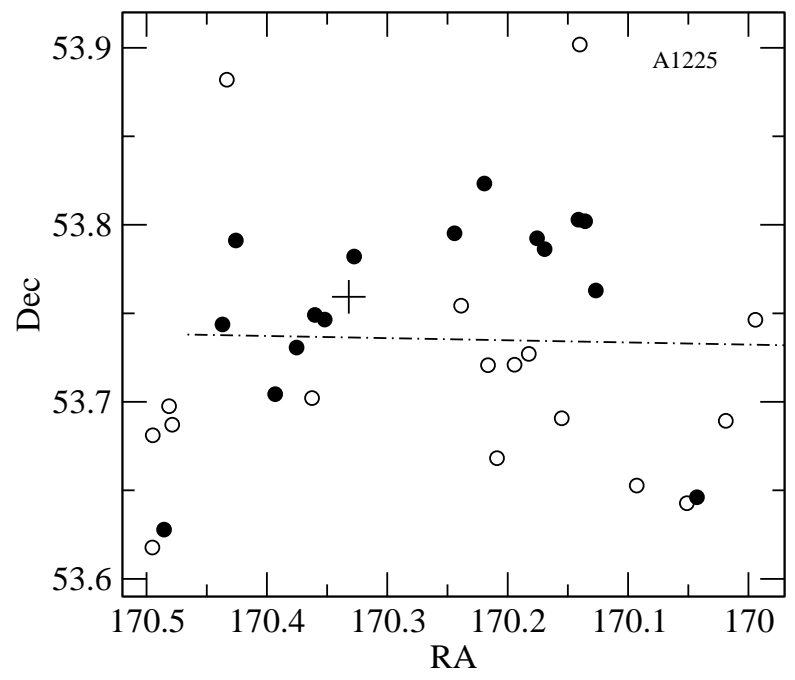

Figure 2: The map of the central region $\left(0.75 R_{A}\right)$ of the rotating cluster $A 1225$. 


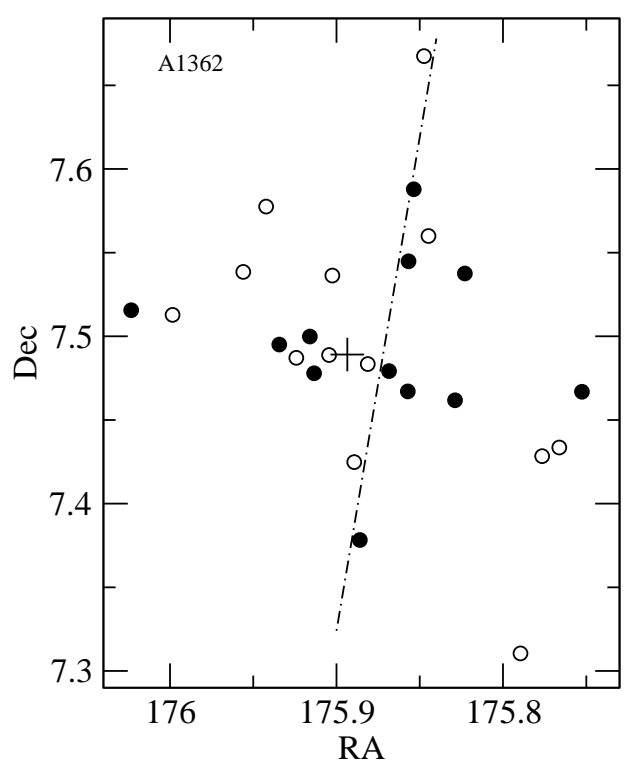

Figure 3: The map of the central region $\left(0.75 R_{A}\right)$ of the rotating cluster $A 1362$. 


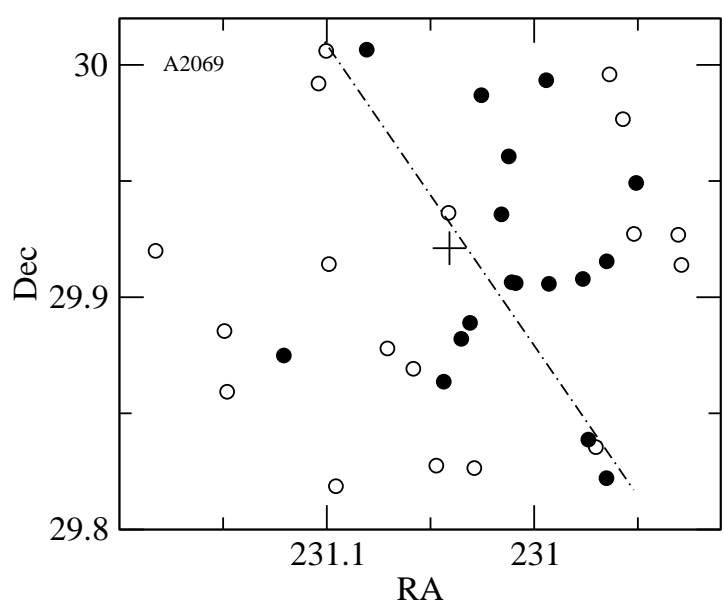

Figure 4: The map of the central region $\left(0.75 R_{A}\right)$ of the rotating cluster A2069. 


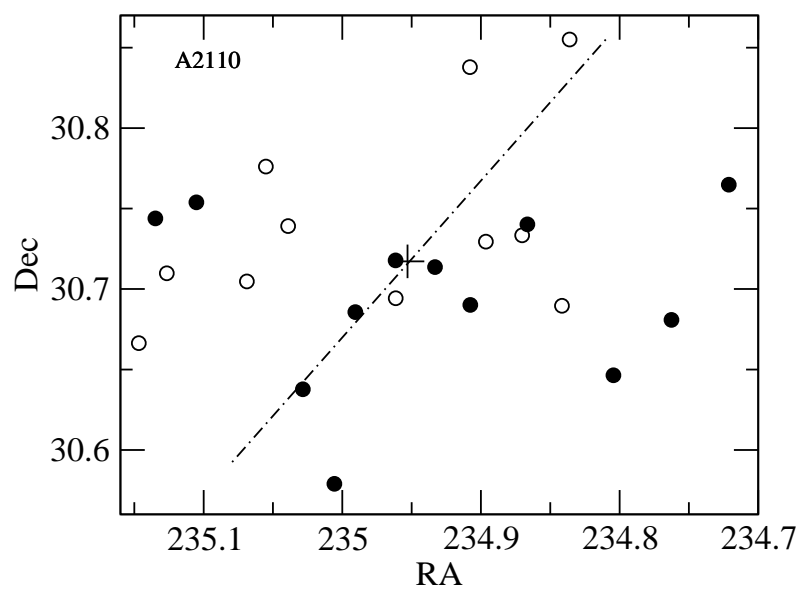

Figure 5: The map of the central region $\left(0.75 R_{A}\right)$ of the rotating cluster $A 2110$. 


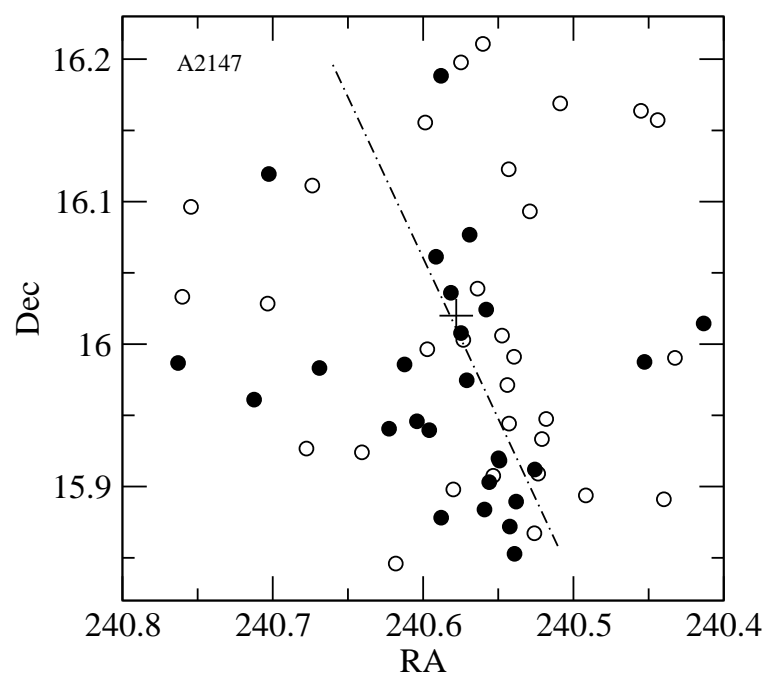

Figure 6: The map of the central region $\left(0.25 R_{A}\right)$ of the rotating cluster A2147. 


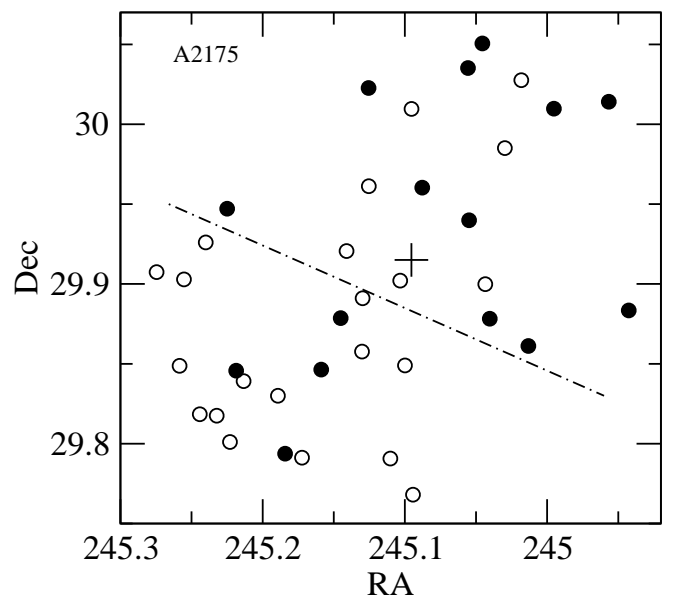

Figure 7: The map of the central region $\left(0.6 R_{A}\right)$ of the rotating cluster $A 2175$. 


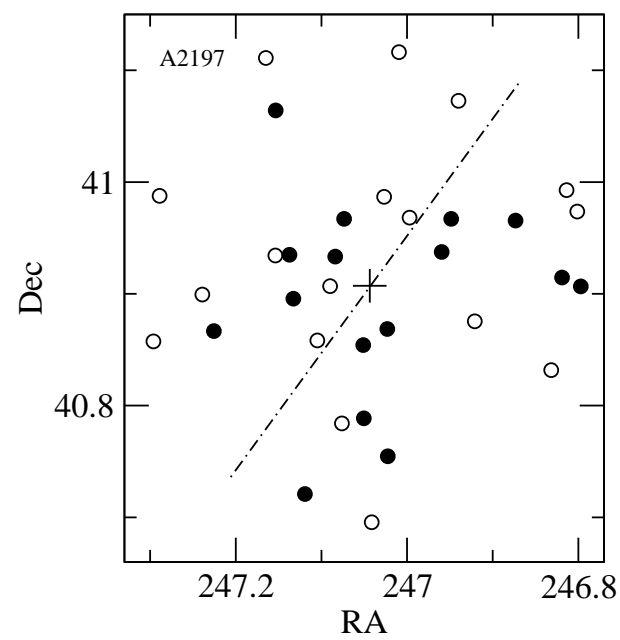

Figure 8: The map of the central region $\left(0.25 R_{A}\right)$ of the rotating cluster A2197. 


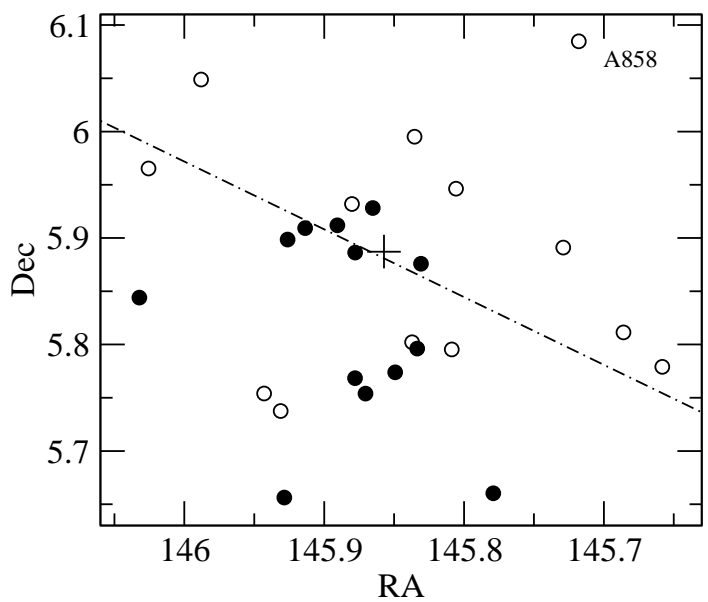

Figure 9: The map of central region $\left(0.75 R_{A}\right)$ of the cluster A858. 


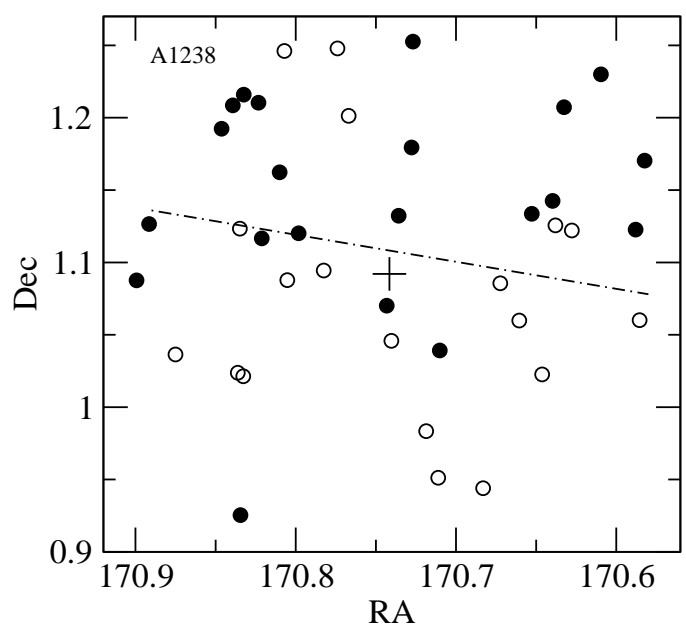

Figure 10: The map of the central region $\left(0.5 R_{A}\right)$ of the rotating cluster A1238. 


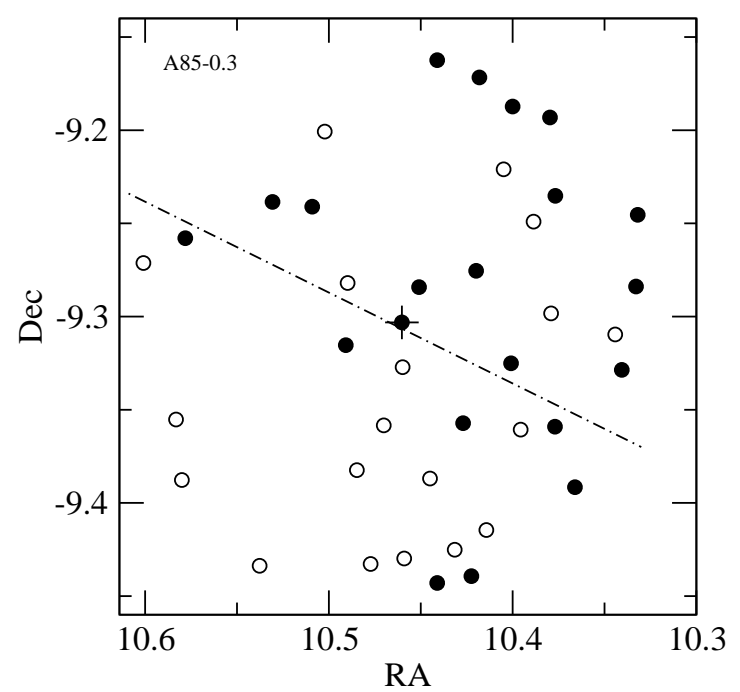

Figure 11: The map of the central region $\left(0.30 R_{A}\right)$ of the rotating cluster A85. 


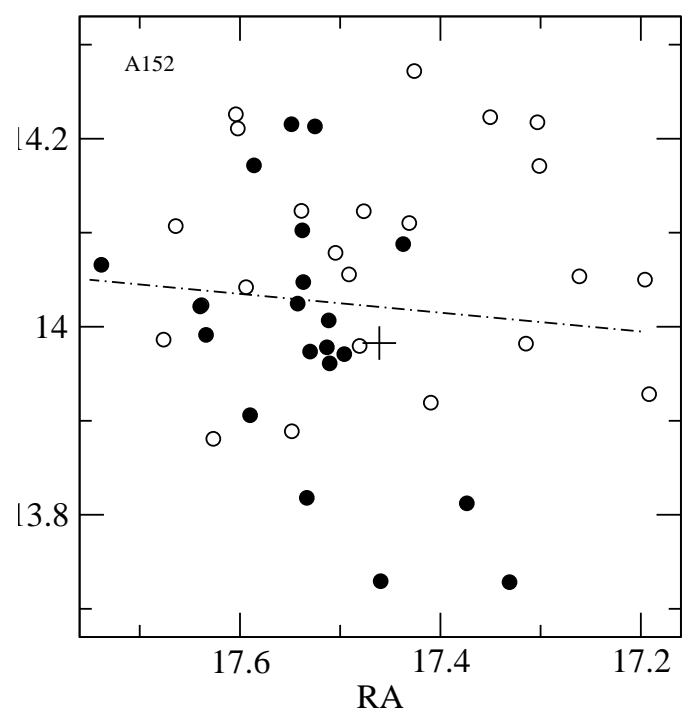

Figure 12: The map of the central region $\left(0.60 R_{A}\right)$ of the rotating cluster A152. 


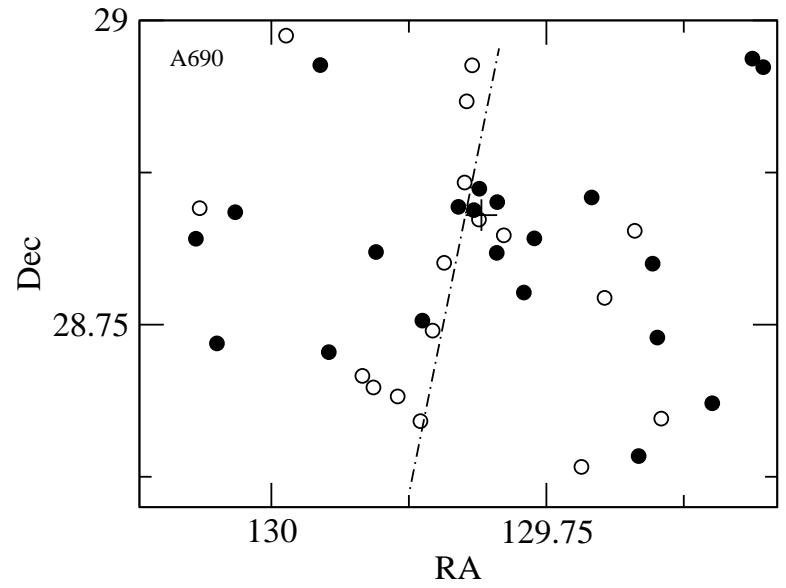

Figure 13: The map of the central region $\left(0.75 R_{A}\right)$ of the rotating cluster A690. 


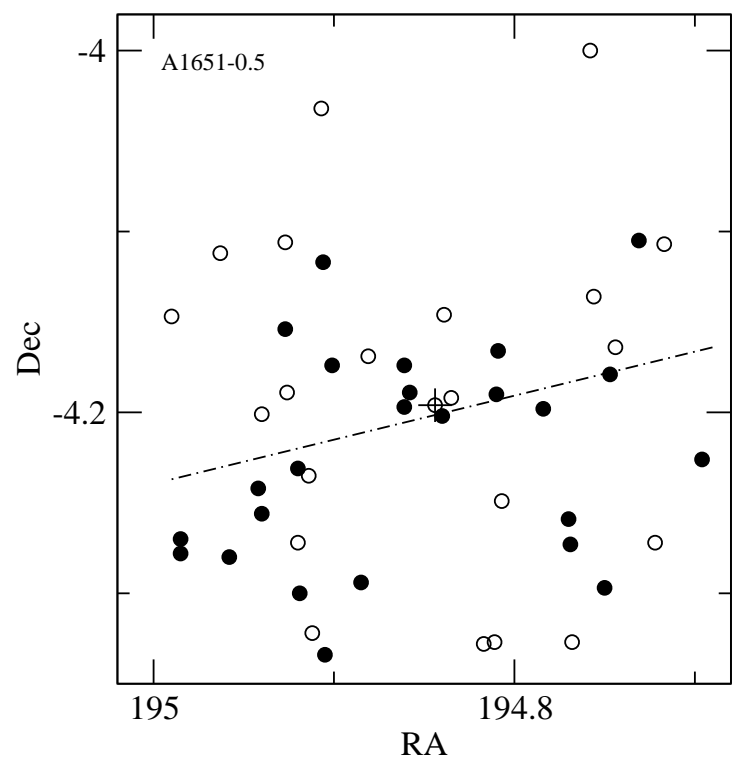

Figure 14: The map of the central region $\left(0.5 R_{A}\right)$ of the rotating cluster A1651. 


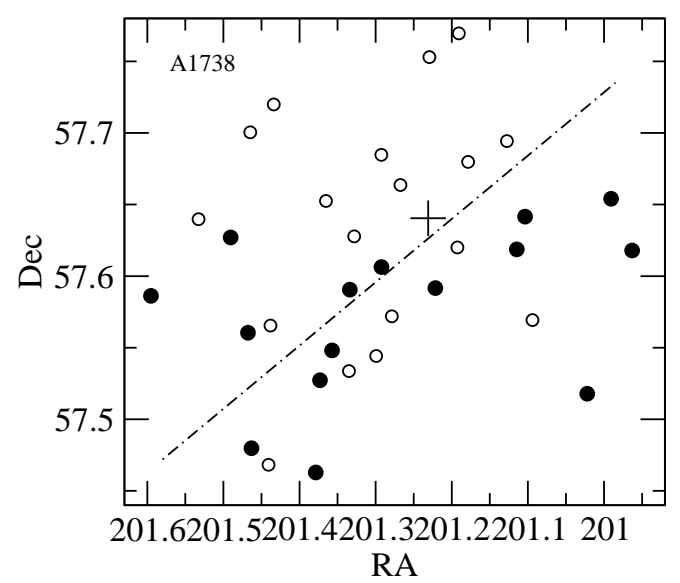

Figure 15: The map of the central region $\left(0.75 R_{A}\right)$ of the rotating cluster $A 1738$. 


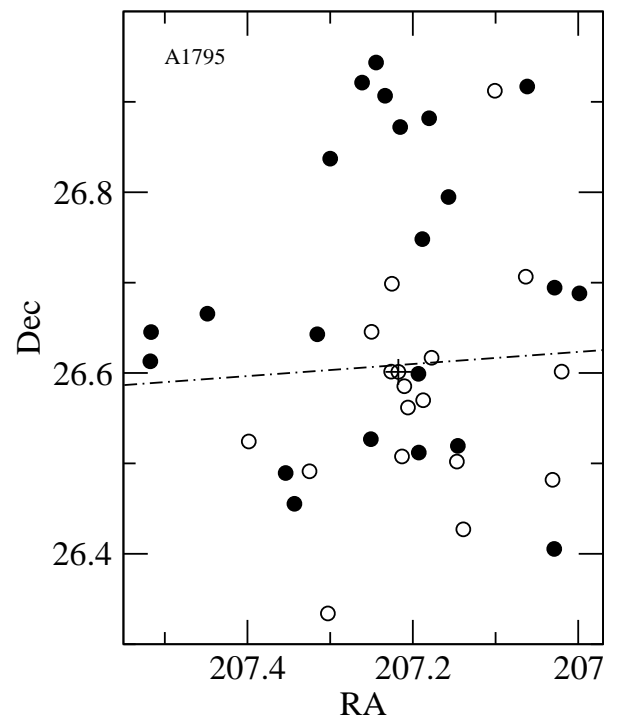

Figure 16: The map of the central region $\left(0.40 R_{A}\right)$ of the rotating cluster $A 1795$. 


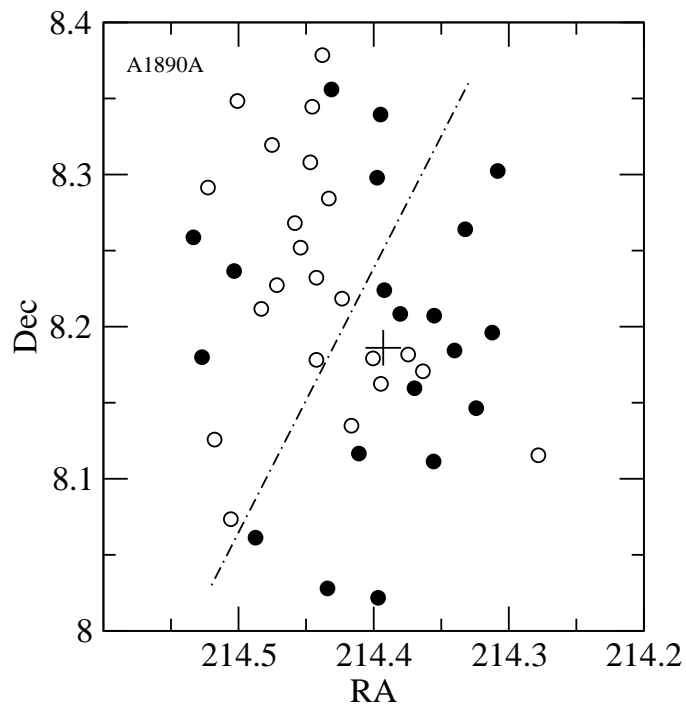

Figure 17: The map of the central region $\left(0.5 R_{A}\right)$ of the rotating cluster $A 1890$. 